\title{
An experimental study on passive charge balancing
}

\author{
K. Sooksood ${ }^{1}$, T. Stieglitz ${ }^{2}$, and M. Ortmanns ${ }^{1}$ \\ ${ }^{1}$ Institute of Microelectronics, University of Ulm, 89081 Ulm, Germany \\ ${ }^{2}$ Laboratory for Biomedical Microtechnology, Department of Microsystem Engineering (IMTEK), University of Freiburg, \\ 79110 Freiburg, Germany
}

\begin{abstract}
This paper presents a simplified analysis of the electrode potential upon mismatched, biphasic stimulation using passive discharge techniques, e.g. by shortening of the electrodes. It turns out that especially for microelectrodes the required shorting intervals become as large as to limit a feasible stimulation interval. If no blocking capacitors can be used due to limited space and the degree of miniaturisation, the passive discharge even imposes severe risks to the surrounding tissue and the electrode.
\end{abstract}

\section{Introduction}

Biomedical implants for functional electrical stimulation (FES), such as the cochlea implant, cardiac pacemaker, and retinal implant have received an increasing interest (Stieglitz and Meyer, 2006; Ortmanns et al., 2007). The principle is to excite a neural reaction upon the transfer of charge into the tissue. Thereby, constant current based stimulators use pulsatile current stimulation via an electrode, which is attached to the human body.

Principally, whenever current is conducted over an electrode into a conducting solution, chemical processes take place at the interface. By applying a large potential over a longer period of time, charge is massively exchanged over the electrode and strong faradaic currents flow which cause electrolysis, $\mathrm{pH}$ change, electrode dissolution as well as tissue destruction (Scheiner and Mortimer, 1990; Liu et al., 2007). In order to avoid these irreversible electrochemical reactions, the stimulating current pulse is typically balanced and biphasic, which ensures that no net charge appears at the electrode after each stimulation cycle and the electrochemical processes are balanced to prevent net dc-currents.

But especially when integrated circuitry is used for the stimulator, due to imperfections of the fabrication process more than $1 \%-5 \%$ of mismatch of the current pulses has to be taken into account. Therefore, measures to achieve charge

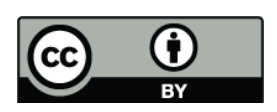

Correspondence to: K. Sooksood (kriangkrai.sooksood@uni-ulm.de) balancing are typically implemented. The most common solution is to insert a large, non-integrated dc blocking capacitor in series with the stimulation electrode, which guarantees that no dc-currents can flow to the electrode over time (Scheiner and Mortimer, 1990; Ortmanns, 2007). Nonetheless, regular discharge of the blocking capacitor is necessary in order to avoid saturation due to dc-current integration and consequently reduced output voltage compliance of the stimulator.

In modern FES applications, where many channels have to be provided concurrently (Ortmanns et al., 2007), dc blocking capacitors can not be realised in the required number due to space limitations. Therefore, the only passive, charge balancing measure is to short the electrodes after the mismatched, biphasic stimulation in order to cancel accumulated charge.

In this work we analyse the dependency of current mismatch, electrode impedance and the resulting steady-state electrode dc-voltage. Within this paper, we model the electrode-electrolyte interface, calculate the net dc-voltage due to imbalance and validate the measurement results by experiments with platinum black electrodes in a $0.9 \%$ saline solution.

\section{Electrode-electrolyte interface model}

A simplified electrode-electrolyte interface can be modelled by an electrolyte resistance in series with the parallel combination of a capacitive path and a charge transfer path (Fig. 1) (Stieglitz, 2004), where $C_{H W}$ and $C_{H C}$ represent the double layer interface capacitor, while $R_{F W}$ and $R_{F C}$ represent the faradaic resistance, of the working and counter electrodes, respectively. $R_{S}$ is the solution spreading resistance, which is beyond others depended on the fluid resistance as well as the electrode properties. Especially for monopolar stimulation, when being compared with the working electrode, the counter electrode has a much larger area. Thus, $C_{H C}$ is assumed to be a rather large capacitance and can therefore be neglected. Generally, in the case of safe operation only $R_{S}$ and $C_{H W}$ are of interest. If the voltage over

Published by Copernicus Publications on behalf of the URSI Landesausschuss in der Bundesrepublik Deutschland e.V. 


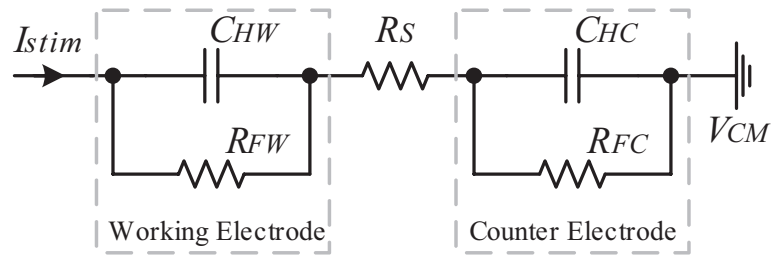

Fig. 1. Simplified electrode-electrolyte interface model.

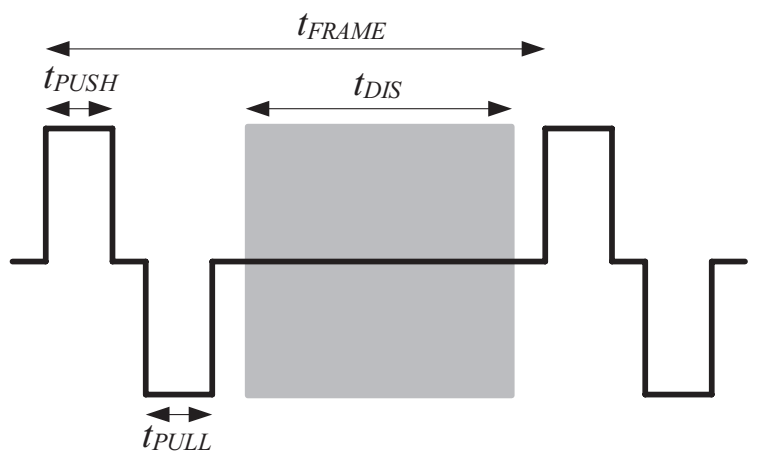

Fig. 2. Timing of a stimulation cycle.

the electrode interface increases permanently to large values, strong faradaic currents flow, which are the source of corrosion and toxicity. This effect is usually not modelled, because it must be avoided by long-term charge balancing (Sit and Sarpeshkar, 2007).

It is important to note that the values of $R_{S}$ and $C_{H W}$ will vary beyond other factors with the material and geometry of the electrodes being used, also with a solution. In this work, two sizes of platinum black electrodes were used with $1000 \mu \mathrm{m}$ and $150 \mu \mathrm{m}$ diameter, respectively. Lumped model parameters $\left(R_{S}\right.$ and $\left.C_{H W}\right)$ have been measured using impedance spectroscopy with $25 \mathrm{mV}$ excitation in a $0.9 \%$ saline solution. Average values for these electrodes were found at $R_{S}=1.2 \mathrm{k} \Omega, C_{H W}=47 \mathrm{nF}$, and $R_{S}=4.7 \mathrm{k} \Omega$, $C_{H W}=18 n F$, respectively.

\section{Electrode potential calculation}

In the following, the derivation of an analytical expression for the steady-state mismatch voltage on an electrode employing passive charge balancing is found. Stimulation pulses in chronic applications are generally symmetric, biphasic and charge balanced (Fig. 2). The duty cycle ( $D C$ ) of this pulse is

$D C=\frac{t_{\mathrm{PUSH}}+t_{\mathrm{PULL}}}{t_{\mathrm{FRAME}}}$

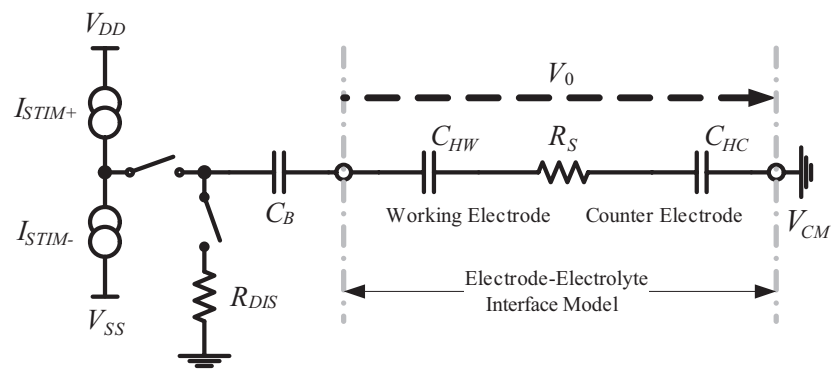

Fig. 3. Biphasic current stimulator with a blocking capacitor.

If there is charge imbalance between the first ("PUSH") and the second ("PULL") current phase, then the average dccurrent of this stimulation pulse is

$I_{D C}=\frac{Q_{\mathrm{PUSH}}-Q_{\mathrm{PULL}}}{t_{\mathrm{FRAME}}}=\frac{I_{\mathrm{STIM}}}{2} \cdot \% M M \cdot D C$

where $I_{\text {STIM }}$ is the stimulation current amplitude, $\% M M$ is the percentage charge mismatch between the push and pull duration, and $D C$ is the duty cycle from Eq. (1). A stimulation current of $\pm 1 \mathrm{~mA}$, a mismatch of $5 \%$ and a duty cycle of $20 \%$, for example, corresponds to an averaged dc-current of $10 \mu \mathrm{A}$ through an electrode.

The discharge period $t_{\text {DIS }}$ in Fig. 2, determines the maximum time for shorting the electrodes or discharging the blocking capacitor to get to the $0 \mathrm{~V}$ reference voltage.

A stimulation circuit with (optional) blocking capacitor $C_{B}$ and discharge switch is shown in Fig. 3. Normally, the size of a blocking capacitor must be large, such that the voltage drop across $C_{B}$ does not significantly decrease the voltage compliance of the stimulator, which is required to drive the electrode impedance. Since also the counter electrode is assumed much larger as the stimulation electrode $\left(C_{H C} \gg C_{H W}\right)$, the time constant of this series connection simplifies to:

$\tau \approx R_{S} \cdot C_{H W}$

From the dc-current in Eq. (2), the mismatch charge per each stimulation cycle, $Q_{M M}$; can be calculated as:

$Q_{M M}=I_{D C} \cdot t_{\mathrm{FRAME}}$

During the discharge period, $t_{\mathrm{DIS}}$, the switch is closed, thus, the blocking capacitor is discharged through $R_{S}$ and $R_{\text {DIS }}$. The charge during this period is:

$Q_{\mathrm{DIS}}=\frac{V_{0}}{R_{S}+R_{\mathrm{DIS}}} \cdot t_{\mathrm{DIS}}$

where $V_{0}$ is the quasi-static electrode potential during the discharge phase. To simplify the calculation, it is assumed that $t_{\text {DIS }}$ is much smaller than $\tau$. Then, the voltage at the capacitor and thus at the electrode remains constant during the discharge period. Hence, Eqs. (4) and (5) are set equal:

$\frac{V_{0}}{R_{S}+R_{\mathrm{DIS}}} \cdot t_{\mathrm{DIS}}=I_{D C} \cdot t_{\mathrm{FRAME}}$ 


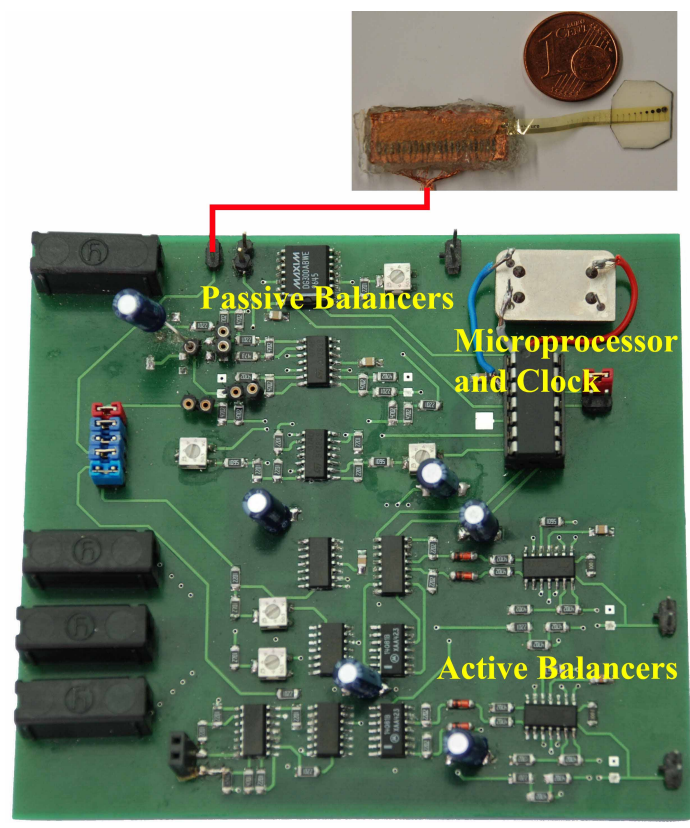

Fig. 4. Implemented printed circuit board with microelectrode.

The electrode voltage can then be calculated to:

$V_{0}=\frac{I_{D C} \cdot t_{\mathrm{FRAME}} \cdot\left(R_{S}+R_{\mathrm{DIS}}\right)}{t_{\mathrm{DIS}}}$

This equation can also be used in the case of stimulator without blocking capacitor (since $C_{B} \gg C_{H W}$ ).

\section{Experiments and discussion}

The given analysis is verified by circuit level simulations using the circuit in Fig. 3, and additionally with experiments using discrete components on a printed circuit board in Fig. 4 in combination with $1000 \mu \mathrm{m}$ and $150 \mu \mathrm{m}$ diameter platinum black electrodes in a $0.9 \%$ saline solution. In addition to blocking capacitor balancing circuit, this circuit board contains other 2 passive balancers and 2 active balancers as well that are not subject of this investigation. The following parameters were used for the experiments: $I_{\mathrm{STIM}}= \pm 500 \mu \mathrm{A}$, a current mismatch of $\% M M=2 \%, C_{B}=22 \mu \mathrm{F}$, while $R_{\mathrm{DIS}}$ was small and could be neglected.

For the pulse timing, in this experiment a high repetition rate of $5 \mathrm{kHz}$ was chosen, with $t_{\mathrm{PUSH}}=t_{\mathrm{PULL}}=50 \mu \mathrm{s}$ and $t_{\text {FRAME }}=200 \mu \mathrm{s}$. These result in charge per phase of $25 \mathrm{nC}$ and $3.18 \mu \mathrm{C} / \mathrm{cm}^{2}$ and $141.5 \mu \mathrm{C} / \mathrm{cm}^{2}$ charge density per phase for $1000 \mu \mathrm{m}$ and $150 \mu \mathrm{m}$ electrodes, respectively. Please note that by extending all timings proportionally, the absolute results of the steady state voltage did not change, since the mismatch current depends only on the duty cycle of the frame (Eq. 2), while the resulting electrode voltage depends on the ratio of the frame and discharge time (Eq. 7).

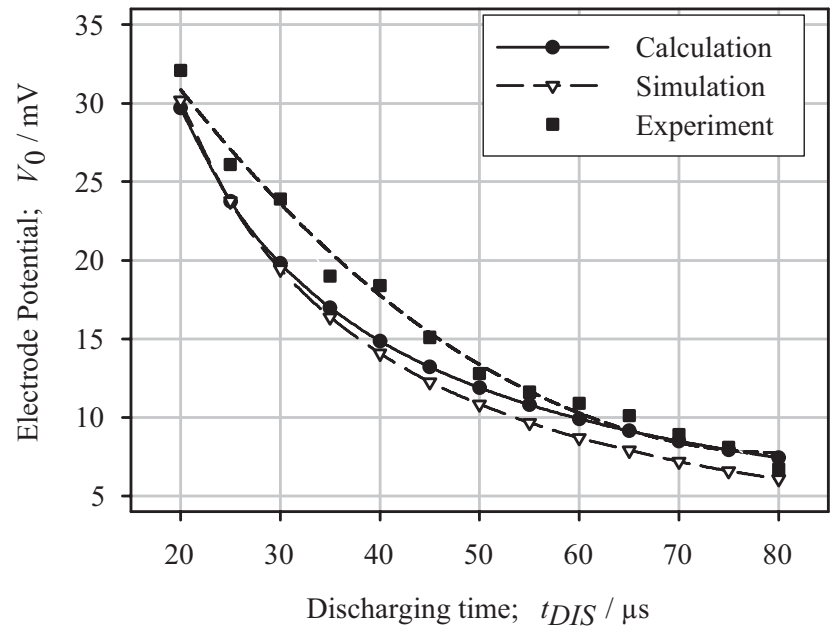

Fig. 5. Electrode potential over discharging time using $2 \%$ mismatch on a $1000 \mu \mathrm{m}$ electrode.

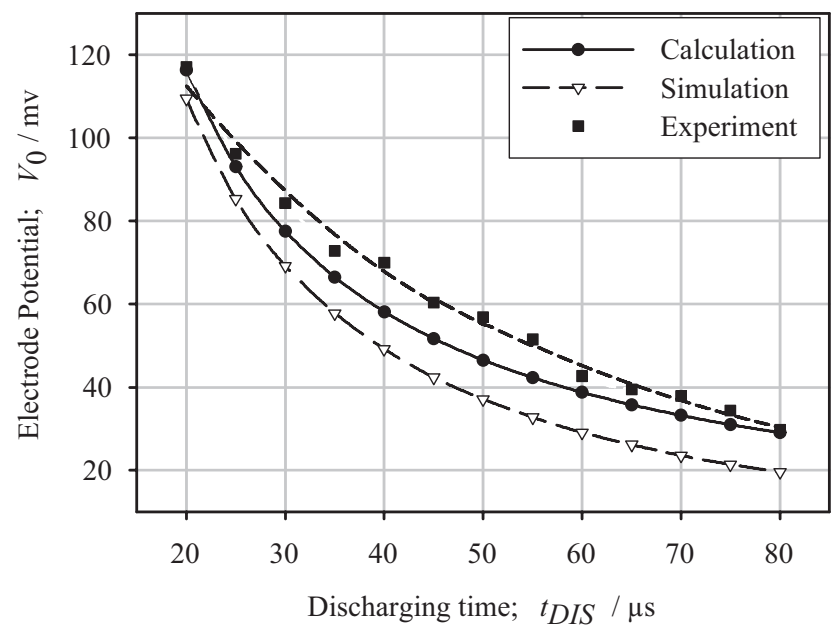

Fig. 6. Electrode potential over discharging time using $2 \%$ mismatch on a $150 \mu \mathrm{m}$ electrode.

Firstly, the $1000 \mu \mathrm{m}$ diameter electrode was used. Figure 5 presents the quasi-static electrode potential after each stimulation and discharge phase. The potential was measured for various discharge times $t_{\text {DIS }}$ and is compared to the result in Eq. (7) and a simulation with the simple circuit in Fig. 3.

Obviously, the analytical model and the experimental simulation and measurement results match well. Differences are caused by the rather small time constant of the electrode impedance and by variations of the same over time. A comparison of the calculation, simulation and the experiment on a smaller electrode with $150 \mu \mathrm{m}$ diameter is given in Fig. 6 . Again, the stimulation parameters were chosen as above.

For illustration of the steady state behaviour, also the transient electrode potential is shown in Fig. 7, where the mismatch caused voltage after each stimulation cycle is seen. 


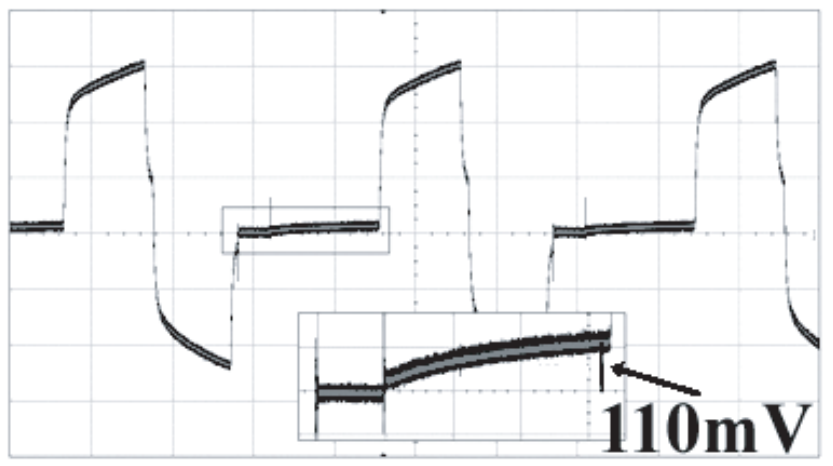

Fig. 7. Excess electrode potential.

From the above results, it is obviously seen that even with the help of blocking capacitors, an electrode potential after stimulation can increase to hundreds of millivolt, if the discharge time becomes very much smaller than the stimulation time. This becomes critical, if for example in multielectrode applications there is no room for external blocking capacitors, and the only charge balancing measure is electrode shortening. In this case, the analytical results are still true, since the series connection of blocking and interface capacitor is dominated by the smaller electrode capacitance.

Furthermore, by decreasing the size of the electrode and thereby increasing the electrode impedance, by using short discharge cycles the quasi-static electrode potential can severely increase (Eq. 7). Consequently, in order to provide a safe stimulation, different ways of charge balancing must be used, for example, active charge balancing (Ortmanns, 2007; Ortmanns et al., 2007; Schuettler et al., 2008).

\section{Conclusions}

This paper presents an electrode potential calculation method for functional electrical stimulation with passive charge balancing using blocking capacitors or electrode shorting. It is shown that a safe value for the discharging time strongly depends on electrode impedance. Thus, when small electrodes are used, a stimulation current mismatch can cause a quasistatic electrode potential exceeding the water window. Thus, either very large safety margins are required or active charge balancing techniques must be used.

\section{References}

Liu, X., Demosthenous, A., and Donaldson, N.: Implantable Stimulator Failures: Causes, Outcomes, and Solution, in: Proceedings of the 29th Annual International Conference of the IEEE Engineering in Medicine and Biology Society, 5786-5789, Lyon, France, 2007.

Ortmanns, M.: Charge Balancing in Functional Electrical Stimulators: A Comparative Study, in: Proceedings of the IEEE International Symposium on Circuits and Systems 2007, 573-576, New Orlean, USA, 2007.

Ortmanns, M., Rocke, A., Gehrke, M., and Tiedtke, H.: A 232Channel Epiretinal Stimulator ASIC, IEEE J. Solid-State Circuits, 42, 2946-2959, 2007.

Scheiner, A. and Mortimer, J.: Imbalanced Biphasic Electrical Stimulation: Muscle Tissue Damage, Annals of Biomed. Eng., 18, 407-425, 1990.

Schuettler, M., Franke, M., Krueger, T. B., and Stieglitz, T.: A Voltage-Controlled Current Source with Regulated Open Circuit Potential for Safe Neural Stimulation, J. Neuroscience Methods, 171, 248-252, 2008.

Sit, J. and Sarpeshkar, R.: A Low-Power Blocking-Capacitor-Free Charge-Balanced Electrode- Stimulator-Chip with Less Than 6 nA DC Error for $1 \mathrm{~mA}$ Full-Scale Stimulation, IEEE Trans Biomed. Circuits and Systems, 1, 172-183, 2007.

Stieglitz, T.: Electrode Materials for Recording and Stimulation, in: Neuroprothetics: Theory and Practice, edited by: Horch, K. W. and Dhillon, G. S., 475-516, World Scientific Publishing, Singapore, 2004.

Stieglitz, T. and Meyer, J. U.: Biomedical Microdevices for Neural Implants, in: BIOMEMS, edited by: Urban, G. A., 71-138, Springer-Verlag, Dordrecht, 2006. 